\title{
Development and characterization of a rotary motor driven by anisotropic piezoelectric composite laminate
}

\author{
S-W Ricky Lee $\dagger$ and H L Li \\ Department of Mechanical Engineering, The Hong Kong University of Science and \\ Technology, Clear Water Bay, Kowloon, Hong Kong
}

Received 2 August 1997, accepted for publication 2 November 1997

\begin{abstract}
A new actuation principle is introduced in this paper to drive a rotary motor by an anisotropic piezoelectric composite laminate. The driving element is a three layer laminated beam with piezoceramics sandwiched between two anti-symmetric composite laminae. By taking advantage of material anisotropy, torsional motion can be induced from in-plane strain actuation. With this structural coupling, a rotary motor can be implemented. In addition to analytical formulation and conceptual design, a prototype has been fabricated. Actual motion was observed in the laboratory to verify the proposed actuation principle. The prototype was characterized for rotating speed, torque, power output, efficiency and stability. The performance of this new piezoelectric motor is discussed in detail.
\end{abstract}

\section{Introduction}

Due to the demand for advanced performance and intrinsic intelligence in modern mechanical systems, the concept of smart materials was introduced in the past decade. The study of intelligent material systems and structures has become a very active research area in engineering nowadays. One of the major characteristics in smart materials is adaptability [1]. In order to achieve this feature, the structural materials must possess sensing and actuation capability. Therefore, the integration of sensing/actuation function and load-bearing capacity is a main subject for smart materials. In the wide spectrum of engineering materials, quite a few of them are considered 'smart' in the literature. Typical examples include shape memory alloy (SMA), piezoelectric ceramics (PZT) and polymer (PVDF), optical fibers, magnetostrictive (MS) and electro/magneto-rheological (ER/MR) materials. Each of the aforementioned smart materials has its own advantages in either sensing or actuation. In particular, piezoelectric ceramics have relatively good performance in both functions. It is also well known that fiber reinforced composites have superior structural properties. Therefore, the hybrids of piezoelectric ceramics and structural composite laminates have a very good potential to form an intelligent material system. The development of such a system has attracted substantial attention from researchers in the area of smart materials. Many studies have been conducted in the past few years. Among them, most efforts were exerted on the theoretical

$\dagger$ E-mail address: RICKYLEE@.UST.HK modeling of mechanical behaviors [2-4]. The actual application of the aforementioned material system is limited so far. Therefore, efforts to identify the appropriate applications of piezoelectric composite laminates are still in demand.

In the literature, piezoelectric materials were used very successfully in acoustics related applications. Recently, piezoceramics are distributed in structures for system monitoring and vibration control [5]. On most occasions, the actuation performance of piezoelectric materials cannot match their counterpart in sensing. In 1973, there was a breakthrough in applying piezoelectric materials to an actuator. Barth [6] successfully made an ultrasonic motor using piezoceramics as the driver. Gromakovskii et al [7] proposed a similar model in 1978. In the ensuing year, Sashida and Kenjo improved the aforementioned two designs and made a wedge-type ultrasonic motor driven by a Langevin vibrator [8]. In the early 80s, Sashida developed a new type of ultrasonic motor by taking advantage of the traveling wave of a stator which is excited by the piezoceramics at the base [8]. The ultrasonic motor earned its name by operating at frequencies in the ultrasonic range $(>20 \mathrm{kHz})$. The main driving mechanism for motion is by structural dynamics and mechanical friction. The advantages of ultrasonic motors include magnetic field immunity, low-speed/high-torque performance, compact size and low noise [9]. Typical applications of ultrasonic motors are the mechanism of auto-focused zoom lenses in video equipment and the driver in watches [10]. Many other applications are under development in industry.

The appearance of ultrasonic motors shows that, even with small deformation, piezoelectric materials still can 


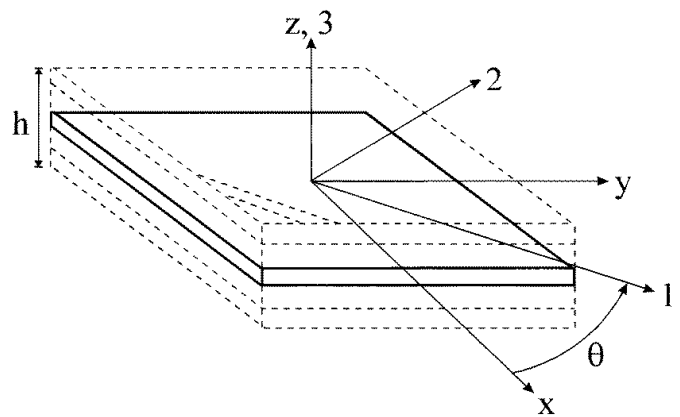

Figure 1. Schematic diagram of laminated structure.

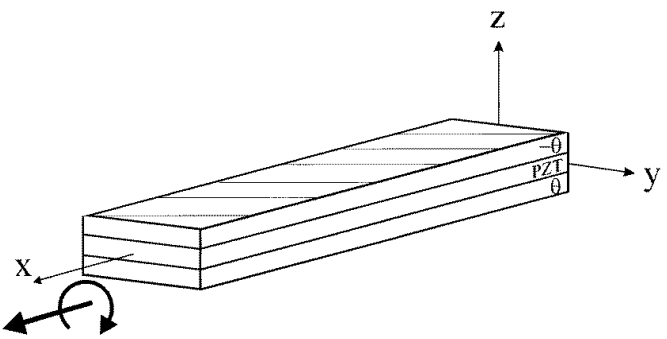

Figure 2. Anti-symmetric laminate with twisting-extension coupling.

actuate and do work as long as there is an appropriate design and mechanism. In this paper, a new principle is introduced to actuate a rotary motor using anisotropic piezoelectric composite laminate. By taking advantage of material anisotropy, a piezoelectric laminated beam can induce torsional motion from in-plane strain actuation [11]. With this structural coupling, a rotary actuator similar to ultrasonic motors can be implemented. The driving element is a three layer laminated beam. A piezoceramic layer is sandwiched between two composite laminae with anti-symmetric configuration. Once the piezo-layer is subjected to an electric field, the whole beam will extend and twist resembling the motion of a screw driver. This motion can be utilized to drive a rotor at one end of the laminate via mechanical friction. In addition to analytical formulation and conceptual design, a prototype has been fabricated. Actual motion was observed in the laboratory to verify the proposed actuation principle. The developed prototype was characterized for rotating speed, torque, power output, efficiency and stability. The performance of this new piezoelectric motor is discussed in detail. The present research will demonstrate a live model for applying the aforementioned smart material system to an actual mechanical device.

\section{Analytical formulation}

Figure 1 shows a general laminated structure composed of a number of layers. Each layer may be anisotropic composite or piezoelectric material. Based on the classical lamination theory [12] and the analysis of a previous study [13], the constitutive relation of a general piezoelectric laminate can be expressed as

$$
\begin{aligned}
\left\{\begin{array}{c}
N_{x} \\
N_{y} \\
N_{x y} \\
M_{x} \\
M_{y} \\
M_{x y}
\end{array}\right\}=\left[\begin{array}{ccccccc}
A_{11} & A_{12} & A_{16} & \vdots & B_{11} & B_{12} & B_{16} \\
A_{12} & A_{22} & A_{26} & \vdots & B_{12} & B_{22} & B_{26} \\
A_{16} & A_{26} & A_{66} & \vdots & B_{16} & B_{26} & B_{66} \\
\cdots & \cdots & \cdots & \cdots & \cdots & \cdots & \cdots \\
B_{11} & B_{12} & B_{16} & \vdots & D_{11} & D_{12} & D_{16} \\
B_{12} & B_{22} & B_{26} & \vdots & D_{12} & D_{22} & D_{26} \\
B_{16} & B_{26} & B_{66} & \vdots & D_{16} & D_{26} & D_{66}
\end{array}\right] \\
\quad \times\left\{\begin{array}{c}
u_{x}^{0} \\
v_{y}^{0} \\
u_{y}^{0}+v_{x x}^{0} \\
-w_{x x}^{\prime} \\
-w_{y y}^{\prime} \\
-2 w_{x y}^{\prime}
\end{array}\right\}-\left\{\begin{array}{l}
N_{x}^{p} \\
N_{y}^{p} \\
N_{x y}^{p} \\
M_{x}^{p} \\
M_{y}^{p} \\
M_{x y}^{p}
\end{array}\right\}
\end{aligned}
$$

where $\left(u^{0}, v^{0}, w\right)$ are mid-plane displacements of the laminate in $(x, y, z)$ directions. Besides, the laminate stiffness matrices, the in-plane force/bending moment resultants and the effective piezoelectric force/moment resultants are given as

$$
\begin{aligned}
& {\left[A_{i j}, B_{i j}, D_{i j}\right]=\int_{-h / 2}^{h / 2} \bar{Q}_{i j}\left[1, z, z^{2}\right] \mathrm{d} z} \\
& {\left[\left(N_{x}, N_{y}, N_{x y}\right),\left(M_{x}, M_{y}, M_{x y}\right)\right]} \\
& \quad=\int_{-h / 2}^{h / 2}\left(\sigma_{x}, \sigma_{y}, \tau_{x y}\right)[1, z] \mathrm{d} z \\
& {\left[\left(N^{p}, N_{y}^{p}, N_{x y}^{p}\right),\left(M_{x}^{p}, M_{y}^{p}, M_{x y}^{p}\right)\right]} \\
& \quad=\int_{-h / 2}^{h / 2}\left[\begin{array}{lll}
\bar{Q}_{11} & \bar{Q}_{12} & \bar{Q}_{16} \\
\bar{Q}_{16} & \frac{\bar{Q}}{22}_{26} & \frac{\bar{Q}_{26}}{\bar{Q}_{66}}
\end{array}\right]\left\{\begin{array}{c}
\bar{d}_{31} \\
\bar{d}_{36}
\end{array}\right\} E_{3}[1, z] \mathrm{d} z
\end{aligned}
$$

respectively, where the superscript $p$ denotes the piezoelectric effect. Note that the terms $\bar{d}_{3 j}$ in (4) are the transformed charge/strain coupling constants of piezoelectric material and $E_{3}$ is the electric field applied to the piezoelectric layer in the thickness direction. For laminates with configuration symmetric to the mid-plane, all $B_{i j}$ terms in (1) will vanish [12]. Otherwise, for asymmetric laminates, $B_{i j}$ may not be zero and will result in various kinds of structural coupling. In particular, if the laminate has an anti-symmetric configuration as shown in figure 2, (1) becomes

$$
\begin{aligned}
\left\{\begin{array}{c}
N_{x} \\
N_{y} \\
N_{x y} \\
M_{x} \\
M_{y} \\
M_{x y}
\end{array}\right\}=\left[\begin{array}{ccccccc}
A_{11} & A_{12} & 0 & \vdots & 0 & 0 & B_{16} \\
A_{12} & A_{22} & 0 & \vdots & 0 & 0 & B_{26} \\
0 & 0 & A_{66} & \vdots & B_{16} & B_{26} & 0 \\
\cdots & \cdots & \cdots & \cdots & \cdots & \cdots & \cdots \\
0 & 0 & B_{16} & \vdots & D_{11} & D_{12} & 0 \\
0 & 0 & B_{26} & \vdots & D_{12} & D_{22} & 0 \\
B_{16} & B_{26} & 0 & \vdots & 0 & 0 & D_{66}
\end{array}\right] \\
\quad \times\left\{\begin{array}{c}
u_{\prime x}^{0} \\
v_{\prime y}^{0} \\
u_{y y}^{0}+v_{\prime x}^{0} \\
-w_{x x}^{\prime} \\
-w_{y y}^{\prime} \\
-2 w_{x y}^{\prime}
\end{array}\right\}-\left\{\begin{array}{c}
N_{x}^{p} \\
N_{y}^{p} \\
N_{x y}^{p} \\
M_{x}^{p} \\
M_{y}^{p} \\
M_{x y}^{p}
\end{array}\right\}
\end{aligned}
$$




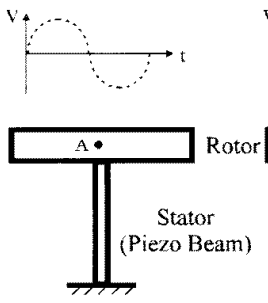

(a) Loose Contact
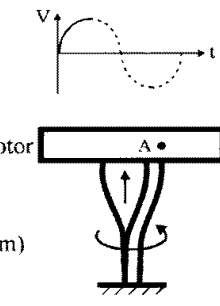

(b) Firm Contact
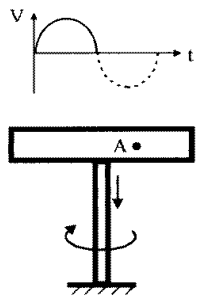

(c) Loose Contact
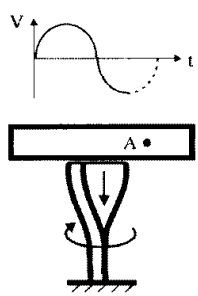

(d) Separate
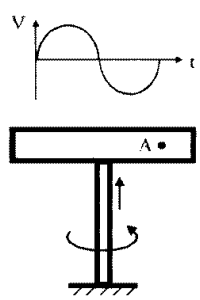

(c) Loose Contact

Figure 3. Schematic diagram for the actuation principle of the rotary motor.

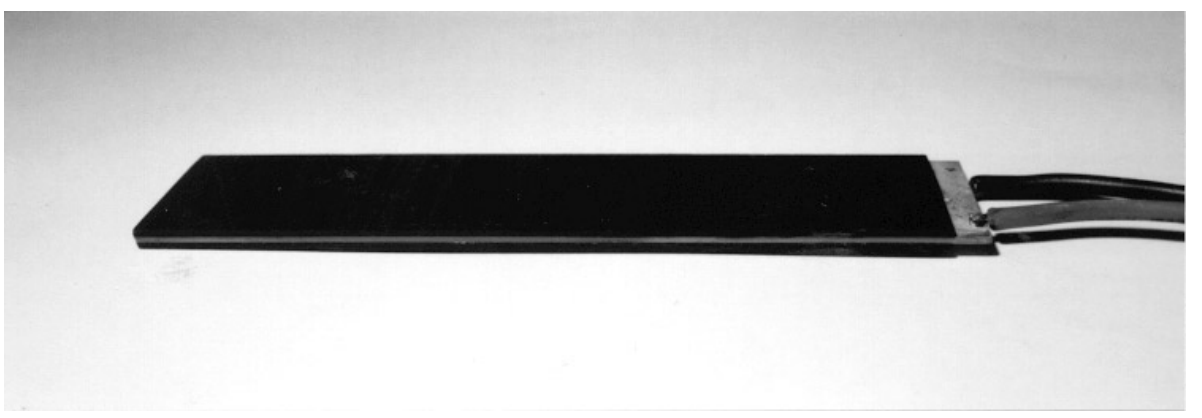

Figure 4. The driving element of the rotary motor: anisotropic piezoelectric composite laminate.

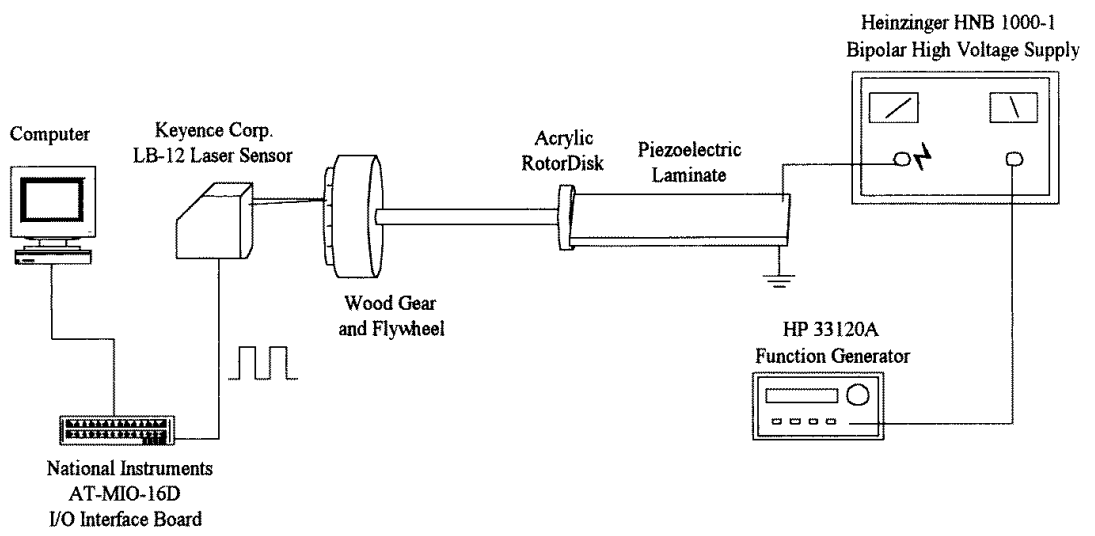

Figure 5. Schematic diagram for the experimental setup.

and twisting-extension coupling will occur [12]. This structural coupling effect can be utilized to drive a rotor via in-plane strain actuation and mechanical friction as described in the following section.

\section{Conceptual design and prototyping}

Based on the aforementioned analysis, a rotary actuator can be implemented by taking advantage of the twistingextension coupling.

In figure 3, the stator is an anisotropic piezoelectric composite laminate with anti-symmetric configuration (see figure 2, termed 'piezo beam' hereafter). One end of this piezo beam is fixed to a rigid foundation while the other end is originally in loose contact with a rotor disk (figure 3(a)).
Once the piezo-layer is subjected to a rising electric field, the whole beam will extend and twist resembling the motion of a screw driver. Similar to a clutch mechanism, the extension of the piezo beam will bring one end into firm contact with the rotor. Due to mechanical friction, the rotor will turn with the end of the piezo beam (figure 3(b)). When the electric field decreases from the peak, the piezo beam will contract and twist backward. However, due to the rotary inertia, the rotor will continue to rotate in the same direction although there is a deceleration from the sliding contact (figure 3(c)). Eventually the piezo beam will separate from the rotor disk (figure 3(d)) and the latter will continue to turn by rotary inertia (figure 3(e)). In such a kind of cycle, the rotor has rotated by a small angle. With this pattern, a continuous rotary motion can be achieved by applying a high frequency cyclic voltage to the piezoelectric laminate. 


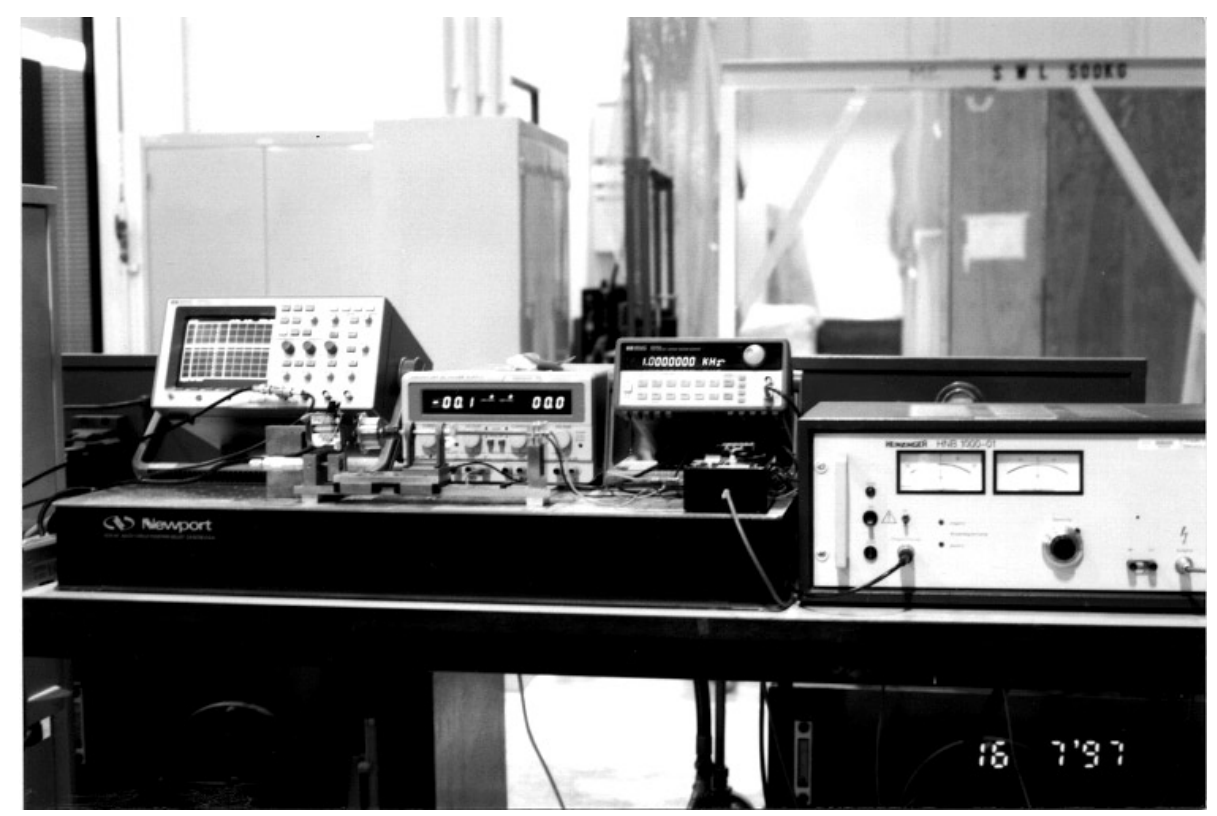

Figure 6. Test rig, equipment and facilities for prototyping.

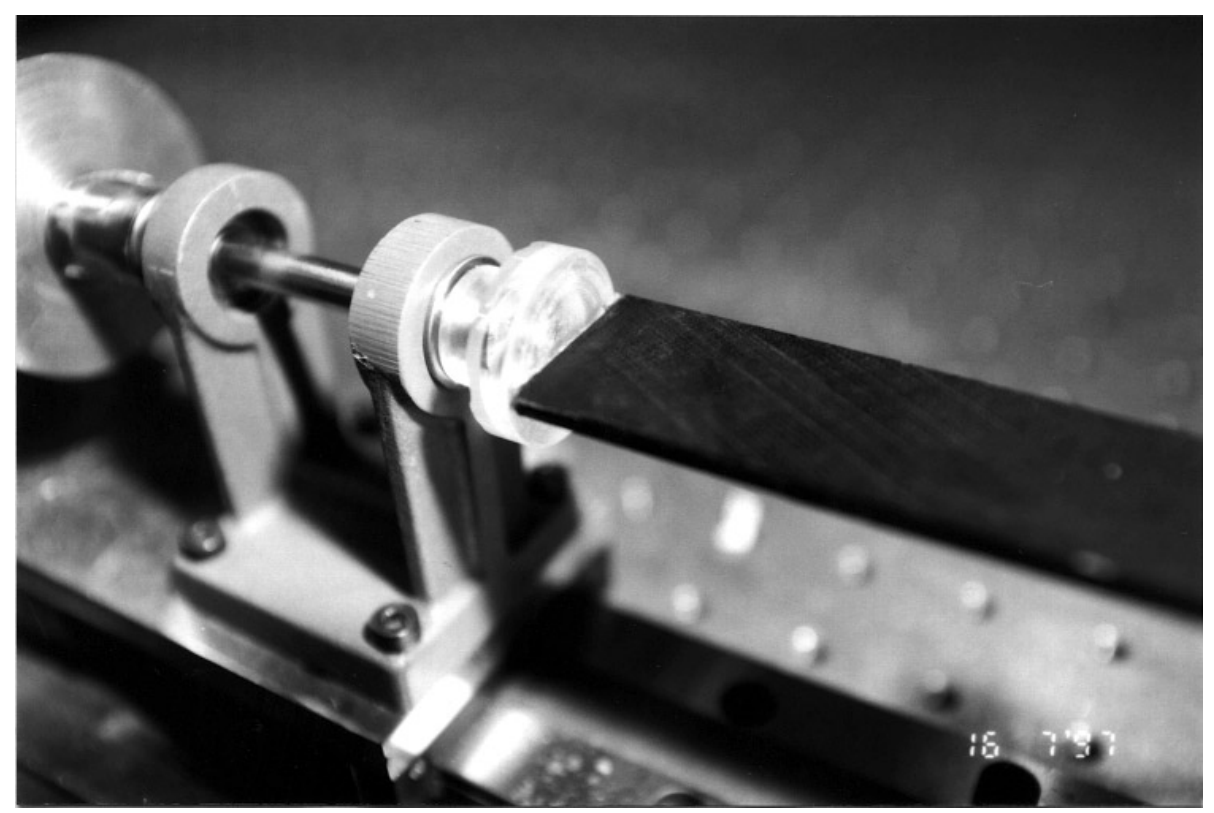

Figure 7. Close-up view for the driving element and the rotor.

During the course of this study, a prototype was fabricated to verify the proposed actuation principle. The driving element was a three-layer composite laminate as shown in figure 4 . The top and the bottom plies were unidirectional AS4/3501-6 graphite/epoxy composites from Hercules. The fiber direction was oriented at $45^{\circ}$ and $-45^{\circ}$ (see figure 1 for $\theta$ ), respectively. The middle layer was made of PZT-5H from Morgan Matroc. The thickness of each layer was $1 \mathrm{~mm}$. The dimensions of the laminated beam were $25 \mathrm{~mm} \times 115 \mathrm{~mm}$. The schematic diagram and the photo picture of the experimental setup are given in figures 5 and 6 , respectively. One end of the piezo beam was engaged in a circular rotor disk as illustrated in figure 7 . The other end was clamped by a rigid fixture. The clamping area was $25 \mathrm{~mm} \times 25 \mathrm{~mm}$. Two leads were soldered to the top and the bottom electrode surfaces of the PZT layer and connected to a high voltage power supply. The power supply was modulated by a function generator at the $\mathrm{kHz}$ range and could output voltage up to 1000 volts. A typical case was actuated at $1.3 \mathrm{kHz}$ with $150 \mathrm{~V}$ amplitude. Figure 8 shows the actual rotary motion of the prototype. Therefore, the proposed actuation principle is verified. More detailed system characterization will be discussed in the next section. 


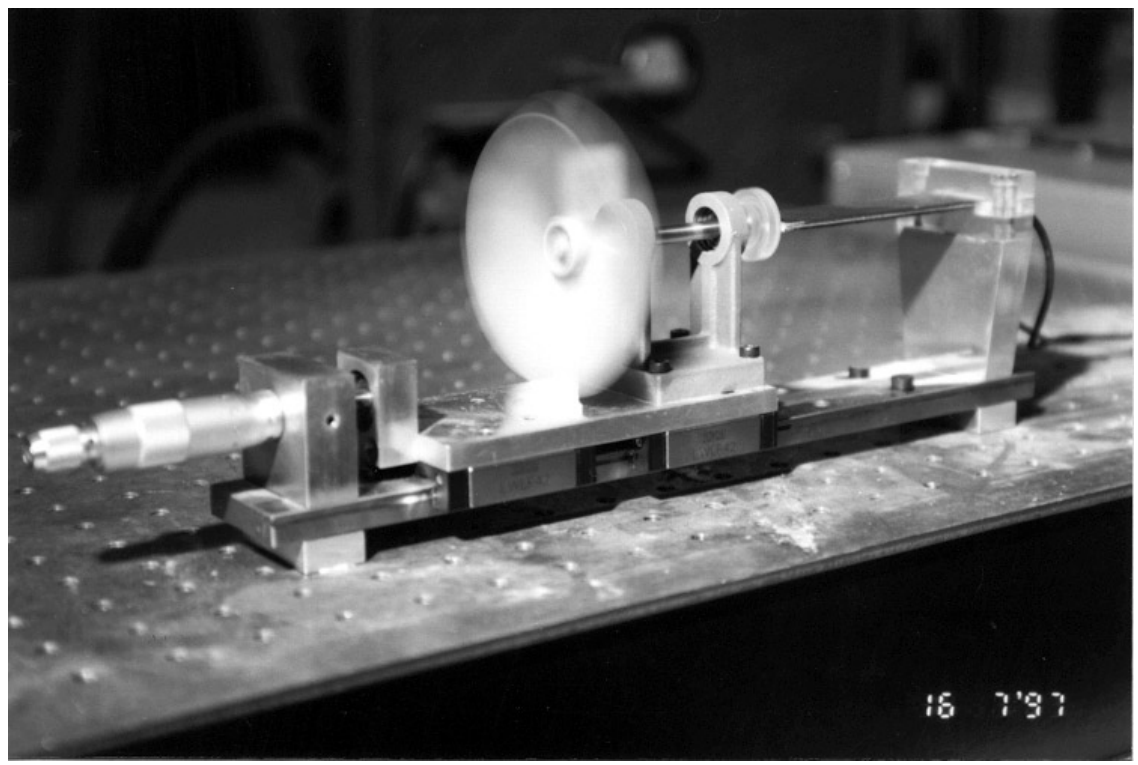

Figure 8. The prototype in motion.

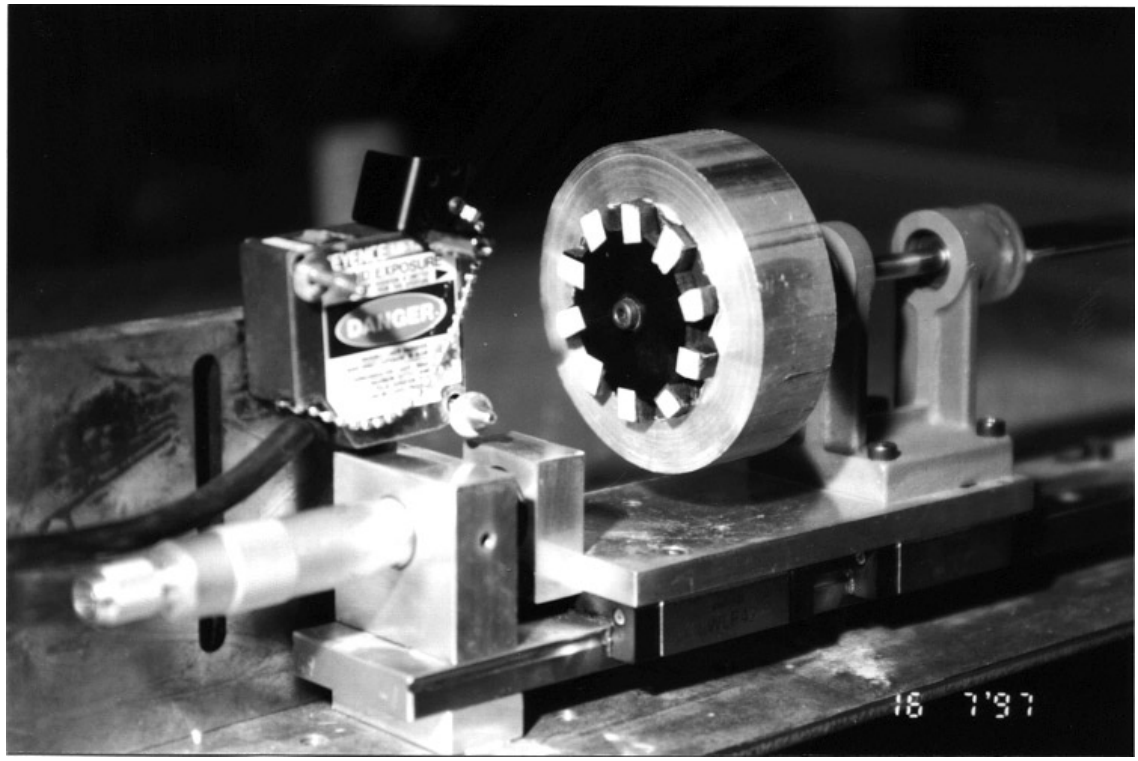

Figure 9. System characterization by flywheel and optical sensor.

Table 1. Specifications of the flywheel set.

\begin{tabular}{lllllc}
\hline Flywheel & Materials & Thickness $(\mathrm{mm})$ & Diameter $(\mathrm{mm})$ & Mass $(\mathrm{kg})$ & $I\left(\mathrm{~kg} \mathrm{~mm}^{2}\right)$ \\
\hline 1 & Cu & 14.37 & 35.50 & 0.1138 & 17.93 \\
2 & Steel & 21.34 & 38.00 & 0.1738 & 31.37 \\
3 & Cu & 19.88 & 50.73 & 0.3196 & 102.8 \\
4 & Al & 22.40 & 76.09 & 0.2652 & 191.9 \\
5 & Steel & 23.40 & 63.64 & 0.5565 & 279.1 \\
6 & Cu & 17.52 & 76.22 & 0.6563 & 476.6 \\
\hline
\end{tabular}

\section{System characterization}

After fabricating the prototype, attempts were made to characterize the system performance. From a previous computational analysis [13], the natural frequency for the first torsional mode of the present piezo beam was $1.5 \mathrm{kHz}$. During the prototyping, it was found that the actual resonance occurred at $1.3 \mathrm{kHz}$. This driving frequency 


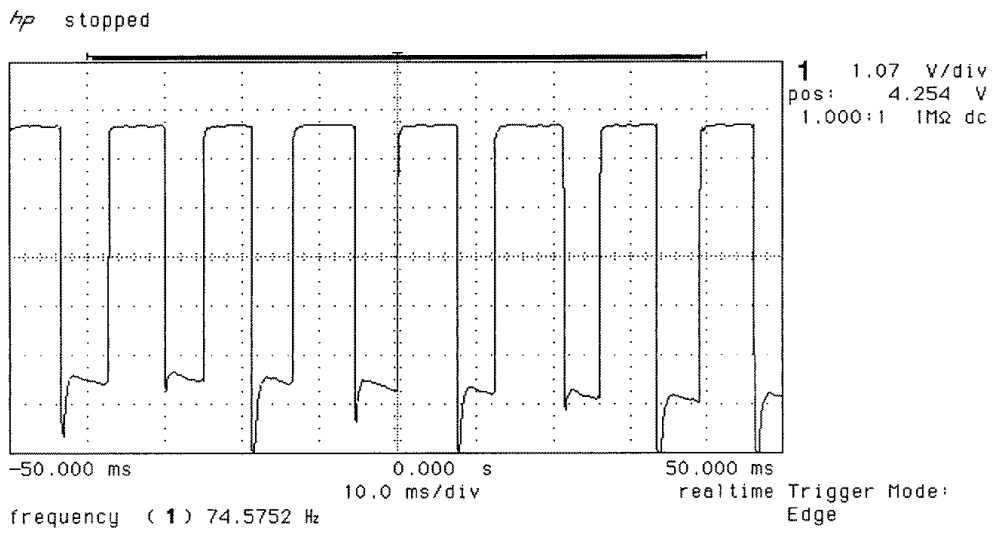

Figure 10. Signal from optical sensor for system characterization.

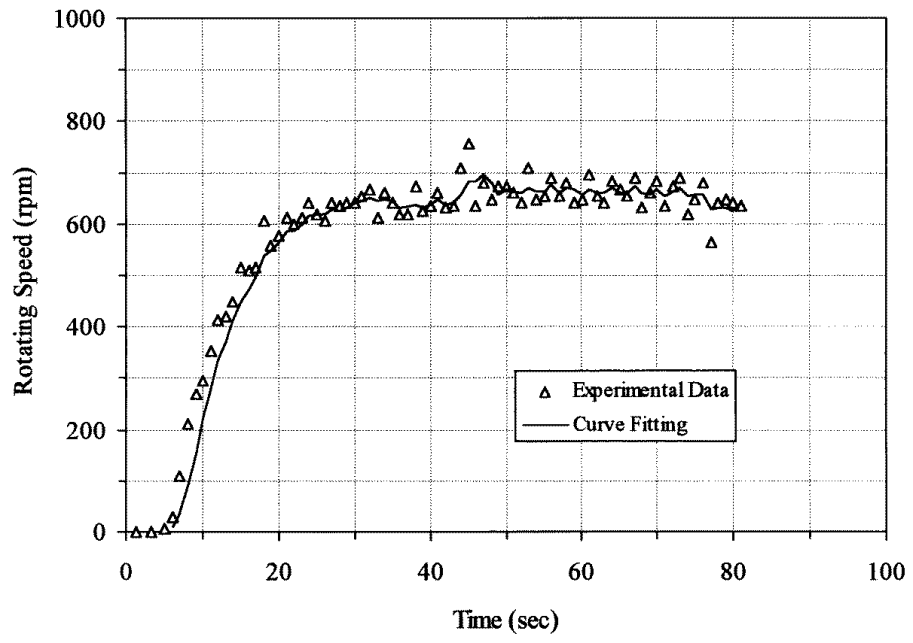

Figure 11. Typical time history of rotating speed response.

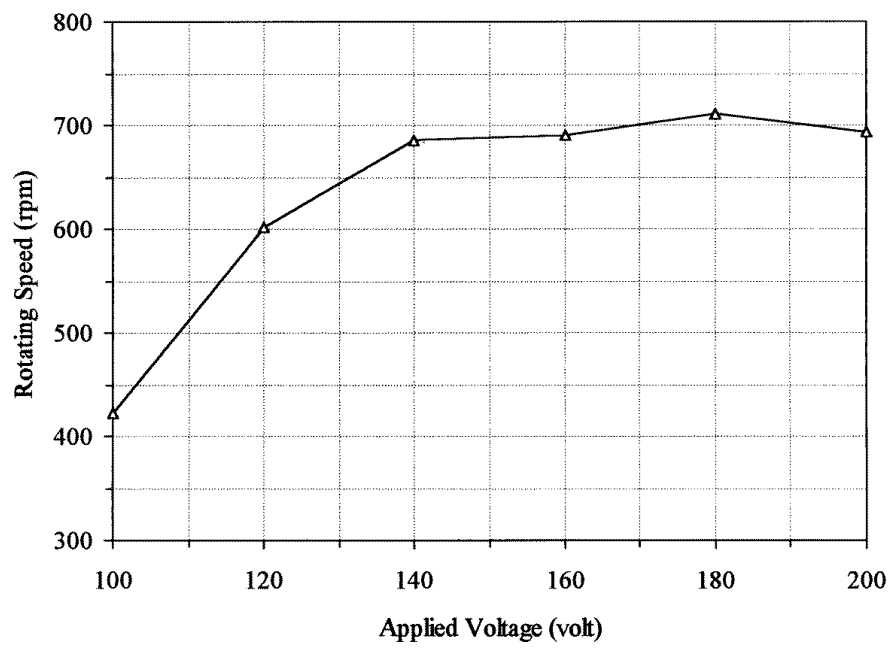

Figure 12. Typical relationship between applied voltage and rotating speed.

was used for the piezo beam in the subsequent system characterization.

The objective of system characterization is to identify the performance of the prototype in rotating speed, torque, power output and efficiency. In order to estimate the above items, an experimental technique was developed. In 


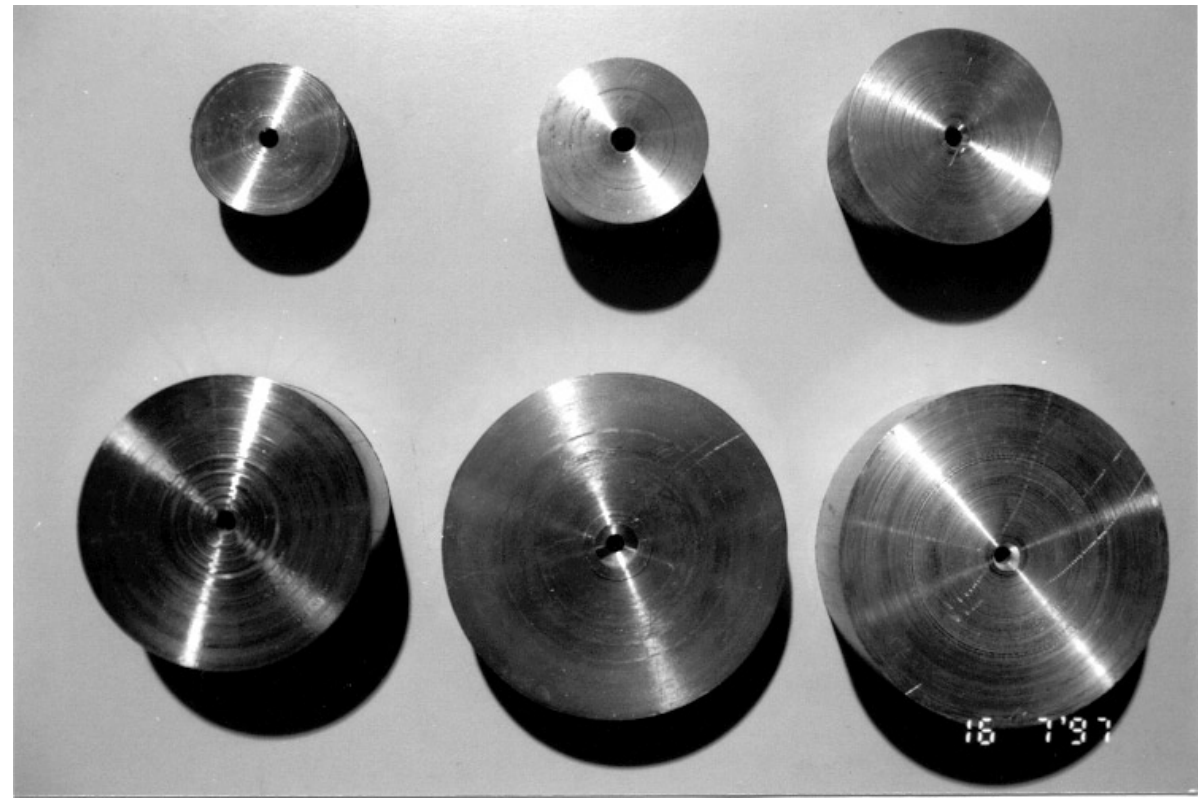

Figure 13. Flywheel set for system characterization.

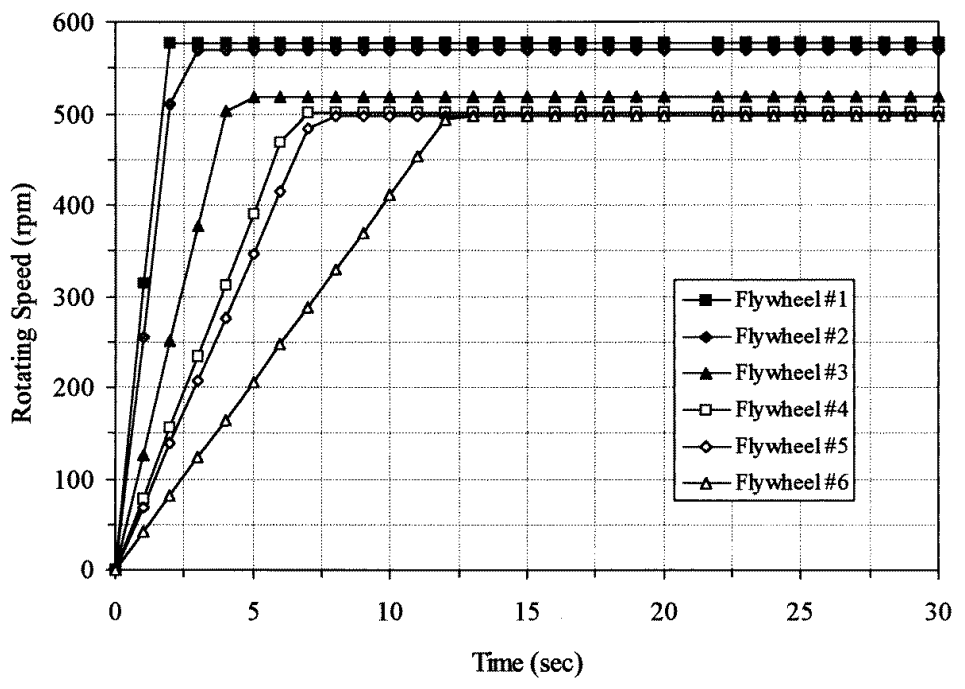

Figure 14. Linearized rotating speed response for torque calculation.

figure 5, a flywheel was installed at the left hand side of the shaft of the rotor. A light wood gear for marking purposes was attached to the flywheel as shown in figure 9. A class IIIB laser displacement sensor was used to detect the gap between teeth of the wood gear. For each gap, a low-true pulse was generated as shown in figure 10. By the pulse counts and the time base, the angular velocity of the rotor could be calculated. In order to automate this process, a personal computer together with a data acquisition board was connected to the laser sensor as shown in figure 5 . The detected signal was processed by commercial software, LabView. The rotating speed of a typical case driven at $150 \mathrm{~V}$ is presented in figure 11 . The moment of inertia of the flywheel was $476.6 \mathrm{~kg} \mathrm{~mm}^{2}$. It is observed that the rotor was accelerated from a stationary state and then reached a steady state with a constant angular velocity. By varying the applied voltage, it was found that the rotating speed of the rotor depends upon the voltage. The relationship between the applied voltage and the steady state angular velocity for the same flywheel as above is presented in figure 12. An approximately linear range between $100 \mathrm{~V}$ and $140 \mathrm{~V}$ (zero-to-peak) was identified. Above $140 \mathrm{~V}$, the angular velocity is saturated and cannot increase any more. Besides, it was found that the rotor could not turn if the applied voltage is below $80 \mathrm{~V}$. For the range between $80 \mathrm{~V}$ and $100 \mathrm{~V}$, the rotary motion was unstable.

In order to characterize the performance of the prototype under different loading, a set of flywheels with various moments of inertia $(I)$ was fabricated as shown in figure 13. Detailed specifications of the flywheel set 


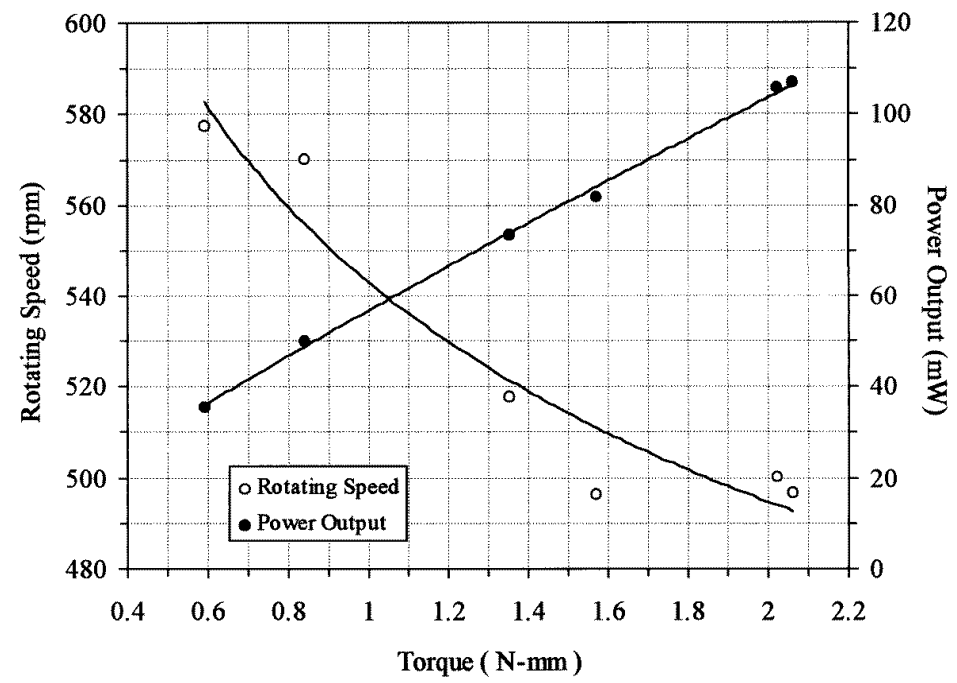

Figure 15. Performance curves of the prototype.

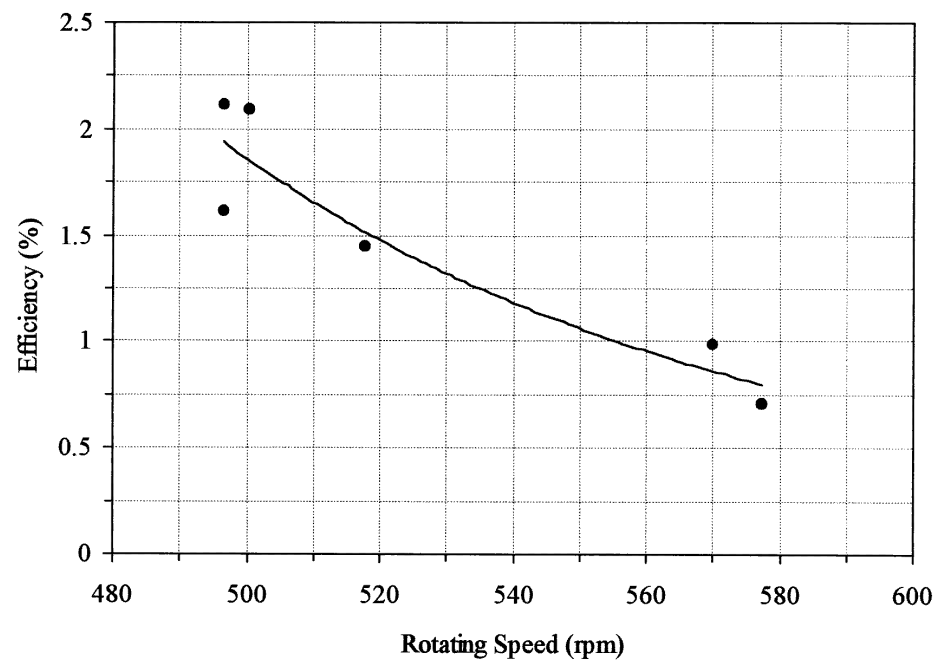

Figure 16. System efficiency against rotating speed.

are given in table 1. Again, the angular velocity could be calculated as before. The torque could be obtained from the definition as

$$
T=I \alpha
$$

where $I$ and $\alpha$ are moment of inertia and angular acceleration, respectively. In figure 11 , it seems that the angular velocity response could be approximated by a bilinear model. For simplicity, such an approximation was implemented for all cases of loading as shown in figure 14 . As a result, the angular acceleration could be calculated from the slope of the ascending part. Consequently, the torque could be estimated. It should be noted that the torque obtained by the aforementioned method is an average quantity instead of an instantaneous one. However, due to the lack of sophisticated equipment such as a dynamometer, this is the best that could be done in the present study.

Once the angular velocity and the corresponding torque were obtained, the power output could be calculated from their product. The results are given in figure 15 . It is observed that the torque declines as the angular velocity increases and the output power grows rather linearly with respect to the torque. These phenomena indicate that this rotary motor has a relatively good low-speed performance. Furthermore, in order to evaluate the efficiency of the prototype system, the input electrical power was calculated by measuring the applied voltage and the corresponding current. The system efficiency is defined as the ratio of mechanical power output to electrical power input. The results are presented in figure 16. It is found that the efficiency is less than $2.5 \%$ and the low-speed performance is better than the high-speed.

In addition to the angular velocity, torque, power output and efficiency, the stability is also a major concern for the performance of a rotary motor. Figure 17 shows the angular velocity response of the largest flywheel (No 6) for a continuous operation of three hours. The prototype had 


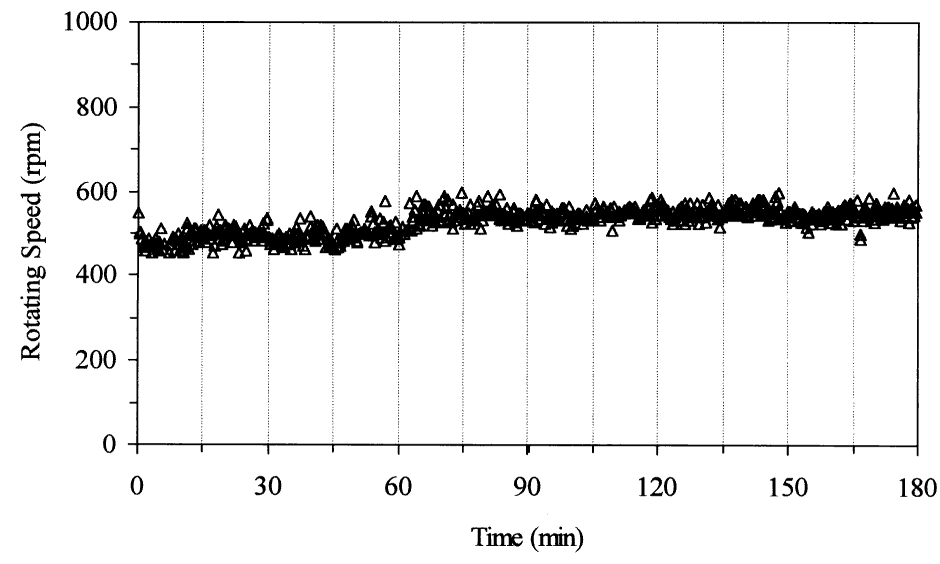

Figure 17. Stability performance of the prototype.

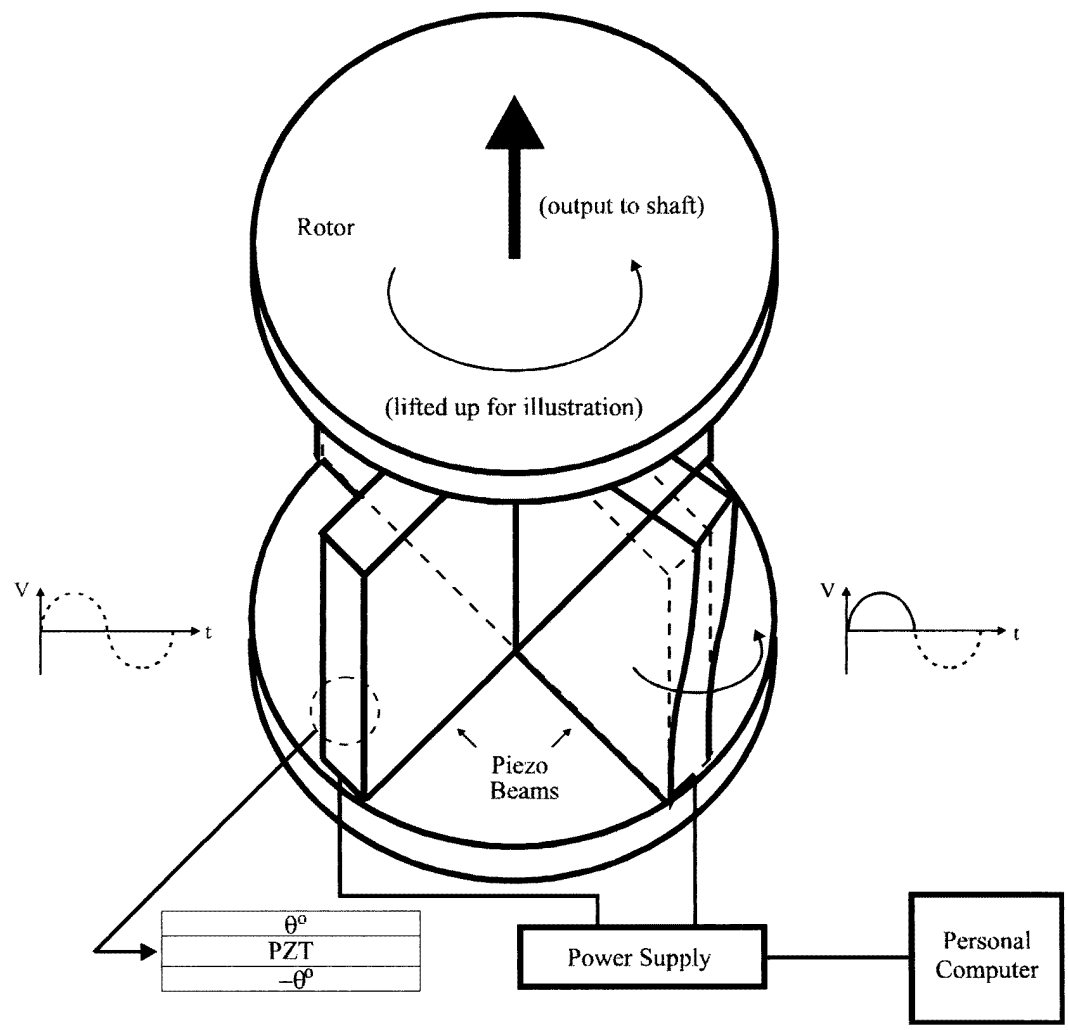

Figure 18. Sophisticated model with multiple driving elements.

no sign of break-down and could maintain a stable rotating speed. Therefore, the stability of this new motor is verified to a certain extent.

\section{Discussion}

The rotary motor developed in this study adopted a new actuation principle which is different from other types of piezoelectric motor. On the other hand, they can be considered as the same category of actuators because the fundamental mechanisms are mechanical vibration and friction. Since the current driving frequency is at $1.3 \mathrm{kHz}$, the present device is not yet a real 'ultrasonic motor' which is usually driven at a frequency higher than $20 \mathrm{kHz}$. However, the resonant frequency can be increased if a piezo beam with smaller dimensions is used. Another alternative is to use composite materials with higher stiffness. It is believed that this motor can be operated at the ultrasonic range with certain modifications in design.

From the design configuration and the results of system characterization, several advantages of the developed motor can be identified. Among them are magnetic field immunity, simple structure for easy maintenance and low cost and good low-speed performance. Besides, this motor 
can be easily programmed to perform intermittent motion. Nevertheless, from the testing data, the system efficiency is rather low. This could be attributed to the nature of actuators driven by mechanical vibration and friction. In order to improve the efficiency, a more sophisticated model with multiple driving elements as shown in figure 18 may be considered. Further design and prototyping are required to prove this concept. Besides, the detailed contact behavior between the piezo beam and the rotor disk has not been investigated in the present study. With further analysis in tribology, the system efficiency may be improved by choosing the appropriate contact geometry and media.

\section{Summary and conclusions}

A rotary motor driven by an anisotropic piezoelectric composite laminate was developed and characterized in this study. The actuation was due to the structural coupling and mechanical friction. The driving element is a three layer laminated beam with piezoceramics sandwiched between two anti-symmetric composite laminae. By taking advantage of material anisotropy, torsional motion can be induced from in-plane strain actuation. With this structural coupling, a rotary motor can be implemented. In addition to analytical formulation and conceptual design, a prototype was fabricated during the course of this study. Actual motion was observed in the laboratory. The proposed actuation principle has been completely verified.

The prototype was characterized for angular velocity, torque, power output, efficiency and stability. The system performance was discussed in detail. It was found that the torque and power output increase while the angular velocity decreases. The advantages of this newly developed piezoelectric motor include magnetic field immunity, simple structure for easy maintenance and low cost and good low-speed performance. In addition, this motor can be easily programmed to perform intermittent motion. However, the system efficiency is rather low for the moment. Some suggestions were made for improvement. Further efforts are required to resolve related issues. In conclusion, the present research has demonstrated a live model of using piezoelectric composite laminates for an actual mechanical device. Although the proposed actuator is still in its infancy, it may lead to many inspiring activities in engineering research.

\section{Acknowledgments}

This study was sponsored by the Research Grant Council of Hong Kong through the grant DAG96/97.EG16 to the Hong Kong University of Science and Technology (HKUST). The authors also wish to acknowledge the equipment support from the Advanced Engineering Materials Facilities of HKUST.

\section{References}

[1] Wada B K and Fanson J L 1990 Adaptive structures $J$. Intell. Mater. Syst. Struct. 1 157-74

[2] Crawley E F and Anderson E H 1990 Detailed models of piezoceramic actuation of beams J. Intell. Mater. Syst. Struct. 1 4-25

[3] Wang B T and Rogers C A 1991 Modeling of finite-length spatially-distributed induced strain actuators for laminate beams and plates J. Intell. Mater. Syst. Struct. 2 38-58

[4] Wetherhold R C and Panthalingal N 1994 Piezoelectric PZT/epoxy composites for controlling torsional motion J. Intell. Mater. Syst. Struct. 5 576-80

[5] Tzou H S 1993 Piezoelectric Shells (Boston, MA: Kluwer)

[6] Barth H V 1973 Ultrasonic driven motor IBM Tech. Disclosure Bull. 162263

[7] Gromakovskii V A et al 1978 On the possibility of using a piezoelectric motor for direct actuation of the drive shaft of a video tape recorder Tekh. Kino Televideniya 5 33-43

[8] Sashida T and Kenjo T 1993 An Introduction to Ultrasonic Motors (Oxford: Clarendon)

[9] Uchino K 1997 Piezoelectric Actuators and Ultrasonic Motors (Boston, MA: Kluwer)

[10] Ueha S and Tomikawa Y 1993 Ultrasonic Motors: Theory and Applications (Oxford: Clarendon)

[11] Lee S-W R and Sun C T 1995 Bending/shearing/twisting sensing and actuation of laminated composite beams with piezopolymer film Intell. Mater. Syst. 10 137-48

[12] Jones R M 1975 Mechanics of Composite Materials (New York: Hemisphere)

[13] Lee S-W R and Chan K H W 1995 Actuation of torsional motion for piezoelectric laminated beams ASME WAM (San Francisco, 1995) Dynamic Response and Behavior of Composites AD-46, pp 139-46 